\title{
A BLADE DESIGN PERFORMANCE BASED OFF-DESIGN LOSS PREDICTION METHOD FOR AXIAL FLOW COMPRESSORS AND CASCADES
}

\author{
Dongrun Wu, Jinfang Teng, Xiaoqing Qiang \\ School of Aeronautics and Astronautics, Shanghai Jiao Tong University, Shanghai, China \\ e-mail: tjf@sjtu.edu.cn
}

ZHONG YANG

AECC Commercial Aircraft Engine Co., Ltd., Shanghai, China

\begin{abstract}
A series of compressor and cascade test recordings are studied to investigate the off-design loss prediction method. The blade design performance is used to predict the off-design loss changing rate at all operating conditions through analytical derivations and statistical correlation studies. The linear correlation between the incidence and a non-dimensional blade loading factor is the foundation of the prediction method. The off-design incidence is normalized using the off-design blade loading factor for different series of blade designs. An analytical method is introduced to predict the off-design blade loading factor based on design parameters and linear correlation. The changing rate of the off-design loss against the blade loading factor is empirically given through statistical analysis. In application, the prediction method can be used to demonstrate the design space of the off-design incidence for a blade series. The modification of the endwall and the rotor tip loss is recommended to give a more accurate prediction in those regions.
\end{abstract}

Keywords: blade design performance, off-design loss, prediction method, axial flow compressor, cascade

\section{Nomenclature}

AVDR - axial velocity $\left(V_{z}\right)$ density ratio $[-], V_{z 2} / V_{z 1}$

$c_{l}, c_{m} \quad-$ blade design lift and off-design loss coefficient [-]

$D_{e q} \quad-$ equivalent diffusion factor $[-]$

$f(\delta) \quad$ - blade loading factor $[-]$

$i \quad-$ incidence $\left[{ }^{\circ}\right]$

$k \quad-$ influence factor of AVDR $[-]$

$m \quad-$ mass flow rate $[\mathrm{kg} / \mathrm{s}]$

$R, R^{2} \quad-\quad$ Pearson correlation coefficient and coefficient of determination [-]

$t_{\max } / c$ - blade maximum thickness to chord ratio [-]

$\beta_{m}, \gamma, \delta-$ mean flow, setting and deviation angle $\left[^{\circ}\right]$

$\eta \quad-$ isentropic efficiency $[-]$

$\kappa \quad-\quad$ angle between blade camber line and meridional plane $\left[^{\circ}\right]$

$\sigma \quad-$ solidity [-]

$\psi \quad-\quad$ static pressure $(p)$ rise coefficient based on density $(\rho)$ and tip rotating speed $\left(U_{t i p}\right)[-],\left(p-p_{t 1}\right) /\left(0.5 \rho U_{\text {tip }}^{2}\right)$

$\omega$

- total pressure $\left(p_{t}\right)$ loss coefficient $[-],\left(p_{t 1}-p_{t 2}\right) /\left(p_{t 1}-p_{1}\right)$

Subscripts: des - design, exp - experimental, max - maximum, min - minimum, norm - normalized, pre - predicted, reg - regression, 1 - at inlet (leading edge), 2 - at outlet (trailing edge). 


\section{Introduction}

Axial flow compressors of aeroengines often operate at off-design conditions during the flight. The performance deterioration at off-design conditions can be serious. Also, the stage matching and the mass flow range depend on the off-design performances. It is desired to make accurate predictions of the off-design loss. In the preliminary design and optimization phase, loss predictions are mostly based on the empirical methods till today (Denton, 1978; Zhang et al., 2017; Banjac and Petrovic, 2018). With modern computing technology, it is feasible to evaluate and optimize the preliminary designs in a short period. However, the generality and accuracy of the previous empirical methods can still be improved. What is more, the Reynolds averaged Navier-Stokes (RANS) based method can make high quality predictions at near design conditions, but has well-known problems in the off-design predictions with flow separations (Leggett, 2018). It is also desired to benefit from both the RANS based design performance predictions and empirical off-design experiences in compressor design procedures.

In previous studies, the influence of the design performance on the off-design loss changing rate was not fully investigated. The blade design performance was mostly used to provide reference values. This means that the changing rate of loss at off-design conditions requires additional tools to solve. Jasen and Moffat (1967), Cetin et al. (1987), Boyer and O'Brien (2002), Schnoes and Nicke (2015) assumed a second or fourth order polynomial variation of loss against incidence. In their methods, the combination of design incidence and loss provided a reference point for the polynomial function. The derivative of the polynomial function that was used to indicate the loss changing rate, was given directly for selected cases (Cetin et al., 1987) or predicted based on additional choke and stall incidence correlations (Jasen and Moffat, 1968; Boyer and O'Brien, 2002; Schnoes and Nicke, 2015). Those critical incidence correlations were dependent largely on blade profiles (Schnoes and Nicke, 2015). And thus, the generality of those methods were compromised.

It was found that the introduction of a non-dimensional blade loading factor can improve the generality of the off-design loss prediction. Swan (1961) found an independent coefficient in the correlation between the changing rate of the off-design loss and the equivalent diffusion factor $D_{e q}$ for DCA (Double Circular Arc) blade profiles at multiple blade spans. König et al. (1996) extended Swan's (1961) method to the applications on NACA65 series and CT (custom-tailored) blade profiles. In the building of the airfoil database, Schnoes and Nicke (2017) also introduced the off-design loss correlation based on $D_{e q}$ in the preliminary design phase. However, in many of the previous applications, the calibration of $D_{e q}$ at off-design condition was only optimized for NACA65 and C.4 series blade profiles (Aungier, 2003). To exclude the empirical calibration for different blade series, this study investigates another non-dimensional blade loading factor $f(\delta)$ in the prediction. Factor $f(\delta)$ is defined as

$$
\begin{aligned}
& f(\delta)=\frac{2}{\sigma}\left[\tan \left(\kappa_{1}+i\right)-\tan \left(\kappa_{2}+\delta\right)\right] \cos \beta_{m} \\
& \beta_{m}=\frac{1}{2} \arctan \left[\tan \left(\kappa_{1}+i\right)+\tan \left(\kappa_{2}+\delta\right)\right.
\end{aligned}
$$

Factor $f(\delta)$ has the potential to be referenced in the off-design loss prediction. The physical meaning of $f(\delta)$ is the lift coefficient of a cascade section without the loss term (Cumpsty, 2004). In a previous study, Wu et al. (2017) introduced a linear correlation between $f(\delta)$ and incidence with AVDR modification. It is feasible to improve the generality of the incidence based off-design loss prediction by incorporating $f(\delta)$ in the method. Also, the linearity between $f(\delta)$ and incidence means that the slope can be evaluated at the design condition and applied at all operating conditions. The influence of the design performance on the off-design loss changing rate can be shown empirically. The off-design performance is predicted with the knowledge of the blade design performance collected from test data, RANS simulations, or design correlations. 
In this study, a blade design performance based off-design loss prediction method is given for axial flow compressors and cascades. Statistical analysis of experimental results is made in building the empirical correlation. The method is validated for both cascades and compressors using another series of experimental results that are not used in the building of the correlation.

\section{Database}

Table 1 and Table 2 list the compressor and cascade test recordings used in this paper respectively. Cases 1 to 8 in Table 1, and case 10 in Table 2 are used to demonstrate the correlations in the prediction method. In Table 1, data are collected from $30 \%$ to $70 \%$ span to reduce the influence of endwalls. The transonic rotors of Stage 35 to 38 and Stage R18/S13 are not discussed in this paper because the losses are fundamentally based on the structure of shockwaves in those rotors. There are 31 blade sections and 482 operating conditions investigated in total. For cascade tests in Table 2, different combinations of the blade design lift coefficient, setting angle, and solidity are considered. There are 79 and 25 different blade settings, 706 and 150 operating conditions in case 10 and 11, respectively. The measured angles are believed to be accurate within $\pm 0.5^{\circ}$. The estimated error of pressure is within $\pm 700 \mathrm{~Pa}$ for compressors. The error margin is relatively large, so the influence of measured accuracy on the prediction method is also discussed in the following sections.

For validation, the predicted values are compared with the test recordings of General Electric (GE) Energy Efficient Engine $\left(E^{3}\right)$ Low Speed Research Compressor (LSRC, case 9), and a series of linear cascade test recordings with 6 percent maximum thickness to chord (case 11). In case 12, three recently designed CT blade profiles in the authors' research group are introduced. The blade profiles of SI, SII, and SIII are shown in Fig. 1. The tests are conducted with compressible inlet flows (inlet Ma from 0.3 to 0.6). They are used to demonstrate the applicability of the current method on the blade profiles other than traditional NACA65 and circular arc airfoils.

Table 1. Compressor test cases

\begin{tabular}{|c|c|c|c|c|}
\hline No. & Test stage & Row type & Blade profile & Reference \\
\hline \hline 1 & TP1493 & Rotor/Stator, two stage & MCA/DCA & Urasek et al. (1979) \\
\hline 2 & Stage 53 & Rotor/Stator & DCA & Osborn et al. $(1978)$ \\
\hline 3 & Stage 55 & Rotor/Stator & DCA/NACA400 & Lewis and Tysl (1974) \\
\hline 4 & Stage 35 & Stator & MCA & Reid and Moore (1978) \\
\hline 5 & Stage 36 & Stator & MCA & Moore and Reid (1982a) \\
\hline 6 & Stage 37 & Stator & MCA & Moore and Reid (1980) \\
\hline 7 & Stage 38 & Stator & MCA & Moore and Reid (1982b) \\
\hline 8 & Stage R18/S13 & Stator & MCA & Lewis et al. $(1974)$ \\
\hline 9 & GE E3 LSRC & Rotor/Stator, four stage & DCA/NACA65 & Wisler (1981) \\
\hline
\end{tabular}

Table 2. Cascade test cases

\begin{tabular}{|c|c|c|c|c|c|c|}
\hline No. & Blade profile & $\left(t_{\max } / c\right) \cdot 10$ & $c_{l} \cdot 10$ & $\gamma$ & $\sigma$ & Reference \\
\hline \hline \multirow{2}{*}{10} & \multirow{2}{*}{ NACA65 } & 1 & $0,4,8,12,15$, & $30^{\circ}, 45^{\circ}$, & $0.5,0.75,1.0$, \\
& & & $21,24,27$ & $60^{\circ}, 70^{\circ}$ & $\begin{array}{c}\text { Herrig } \text { et al. } \\
(1.25,1.5\end{array}$ & $(1951)$ \\
\hline 11 & NACA65 & 0.6 & $4,8,12$ & $60^{\circ}, 70^{\circ}, 75^{\circ}$ & $0.75,1.0,1.5$ & Emery (1957) \\
\hline 12 & CT (SI/SII/SIII) & $\approx 0.85$ & - & $\approx 20^{\circ}$ & 1.5 & - \\
\hline
\end{tabular}




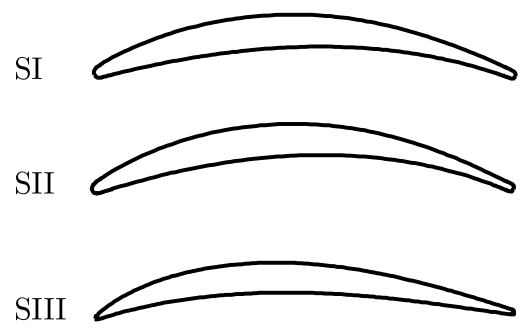

Fig. 1. CT blade profiles in the authors' research group

\section{Building of the prediction method}

The prediction of the off-design total pressure loss $\omega$ is firstly written in an incidence based form

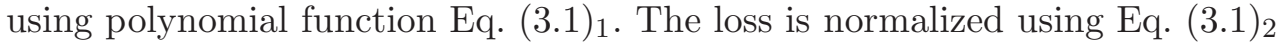

$$
\begin{aligned}
& \omega=c_{m}\left|i-i_{\text {des }}\right| n+\omega_{\text {des }} \\
& \frac{\omega}{\omega_{\text {des }}}=\left|\sqrt[n]{\frac{c_{m}}{\omega_{\text {des }}}}\left(i-i_{\text {des }}\right)\right|^{n}+1=\left|\left(i-i_{\text {des }}\right)_{\text {norm }}\right|^{n}+1
\end{aligned}
$$

where $n$ is a positive real number. Figure 2 gives a normalized plot of the off-design loss against incidence for compressors (a) and cascades (b) when $n$ equals to 3 . The solid lines show the regression values of the data points. Figure 3 shows the influence of $n$ on the mean error (a) and the root mean square error (RMSE) (b) of the polynomial regression. When $n$ equals to 3 , the error is relatively small for both compressors and cascades. For brevity, the polynomial regression results are plotted for $n$ equal to 3 .
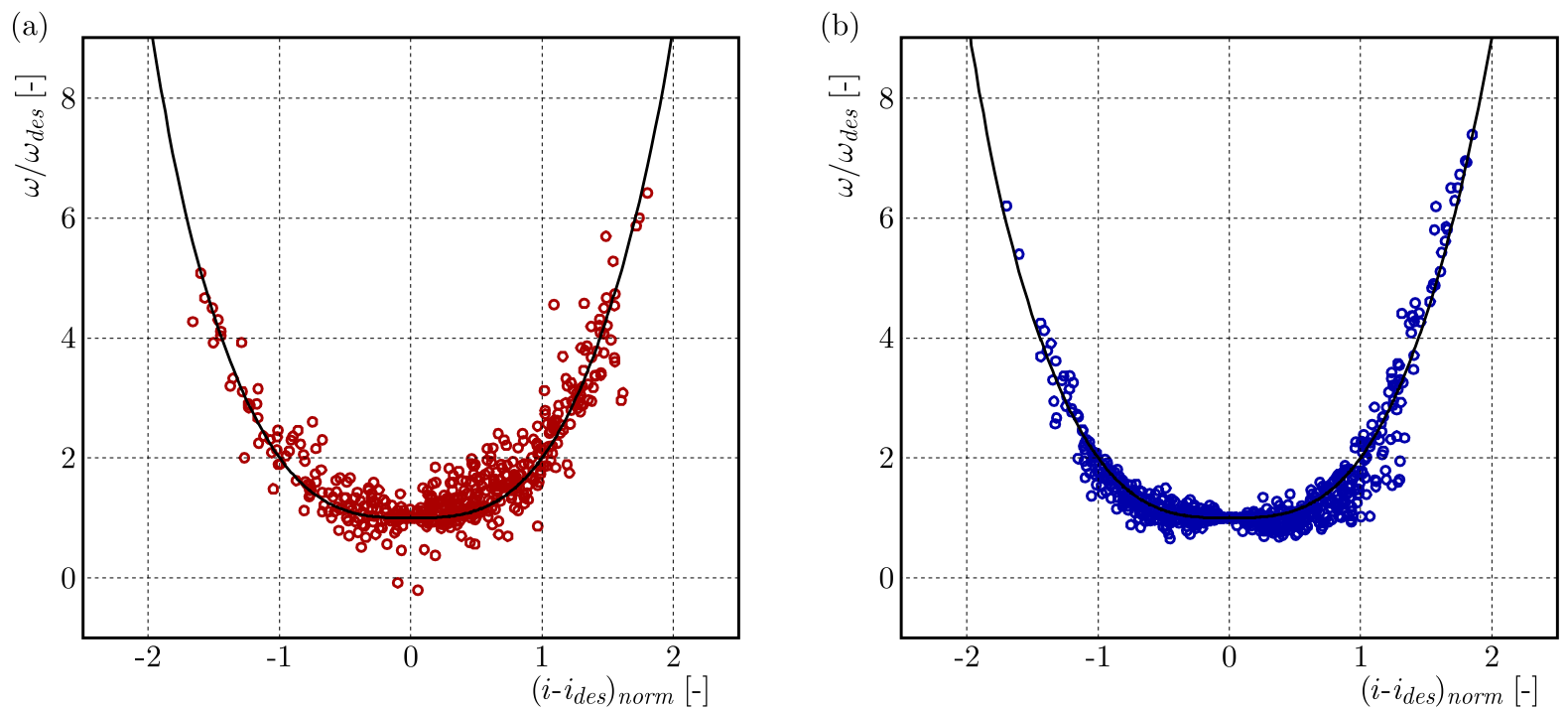

Fig. 2. Normalized plot of the off-design loss against incidence: (a) LHS compressors, (b) RHS cascades

Before transformation of Eq. $(3.1)_{2}$, a correlation between incidence and $f(\delta)$ with AVDR modification is introduced in Fig. 4. The incidence and $f(\delta)$ are normalized using their maximum and minimum values

$$
i_{\text {norm }}=\frac{i-i_{\min }}{i_{\max }-i_{\min }} \quad f(\delta)_{\text {norm }}=\frac{f(\delta)-f(\delta)_{\min }}{f(\delta)_{\max }-f(\delta)_{\min }}
$$

The linearity between the incidence and $f(\delta)$ is significant. The linear regression statistics shows that the linearity is slightly better for cascade tests. In the definition of $f(\delta)$, the influence 

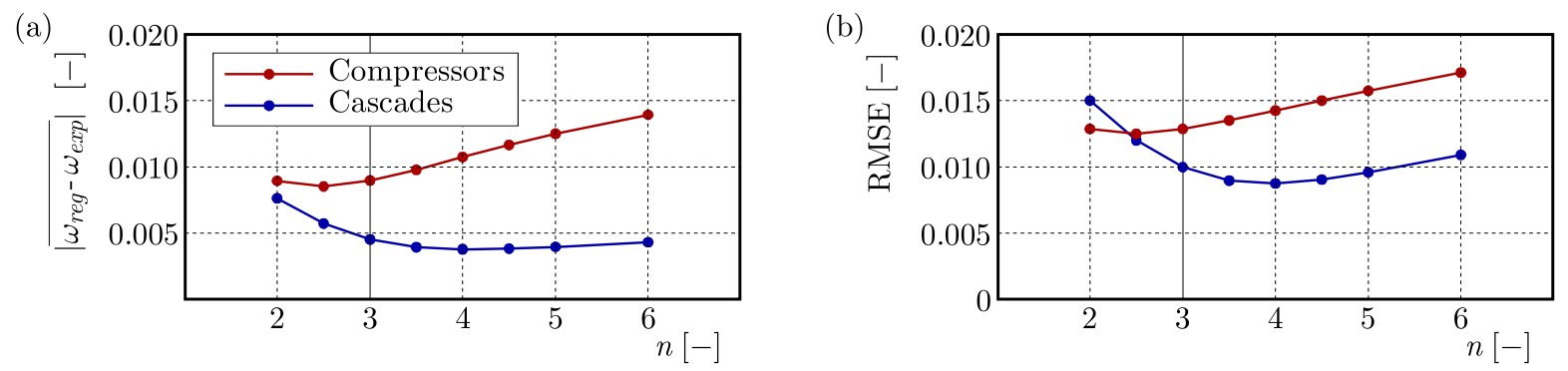

Fig. 3. Influence of $n$ on the mean error (a) and the RMSE (b) of the polynomial regression

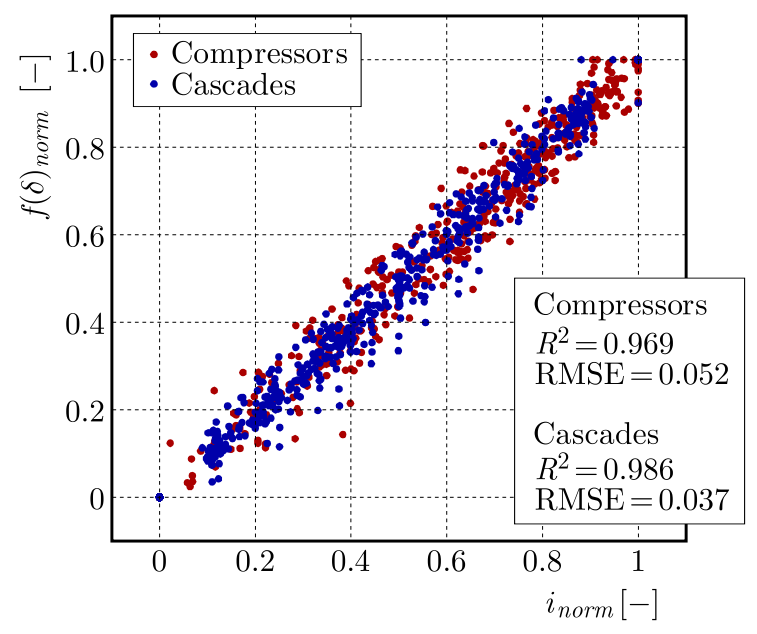

Fig. 4. Normalized plot of $f(\delta)$ against incidence

of the axial velocity ratio is not considered. The AVDR modification is introduced to set all test results to the standard unity axial velocity ratio condition. The experimental deviation angle $\delta_{\text {exp }}$ is modified using the method originally proposed by Pollard and Gostelov (1967)

$$
\delta=\delta_{\text {exp }}-k(1-\mathrm{AVDR})
$$

where $k$ is the influence factor of AVDR. For cascades, $k$ equals 10 according to Pollard and Gostelov (1967). For compressors, it is assumed that the Pearson correlation coefficient between AVDR and the linear regression error of $f(\delta)$ equals 0 . The purpose is to reduce the correlation between the linear regression error and AVDR statistically. The following Pearson correlation equation is solved

$$
R\left(f(\delta(k))-\frac{d f(\delta(k))}{d i} i, \mathrm{AVDR}\right)=0
$$

The solution is $k$ being equal to 7.6 for compressors.

The sensitivity of measured angle accuracy on $f(\delta)$ is also examined. The difference of $f\left(\delta+0.5^{\circ}\right)$ and $f\left(\delta-0.5^{\circ}\right)$ is calculated and normalized (using the maximum and minimum value of $f(\delta)$ for the same blade section). The normalized difference between $f\left(\delta+0.5^{\circ}\right)$ and $f\left(\delta-0.5^{\circ}\right)$ is smaller than 0.14 for all test conditions, which is much larger than the RMSE in Fig. 4. The estimated error margin is generally conservative. The linear correlation is significant with the actual test error.

Figure 5 shows that the linear correlation between the incidence and $f(\delta)$ also exists for CT blade profile cascades with compressible inlet flows. The SI and SII blades are tested at four different inlet Ma conditions from 0.3 to 0.6. The relative change of $f(\delta)$ is less than $5 \%$ for different inlet Ma numbers. The variation is relatively small within a certain Ma range. Figure 6 


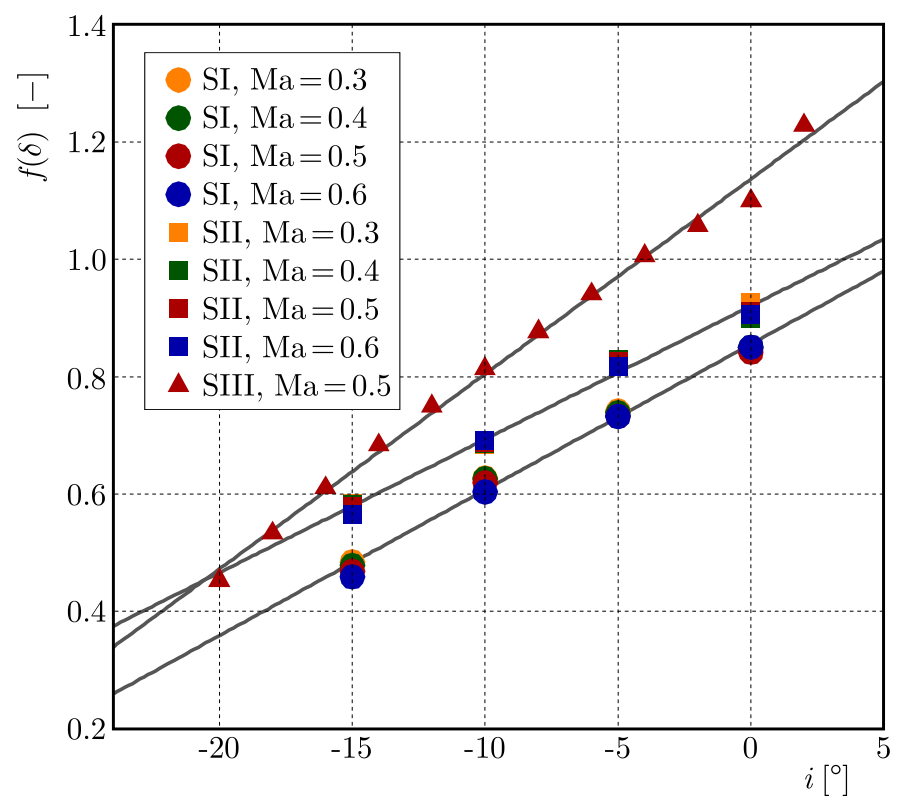

Fig. 5. Plot of $f(\delta)$ against incidence for CT blade profile cascades

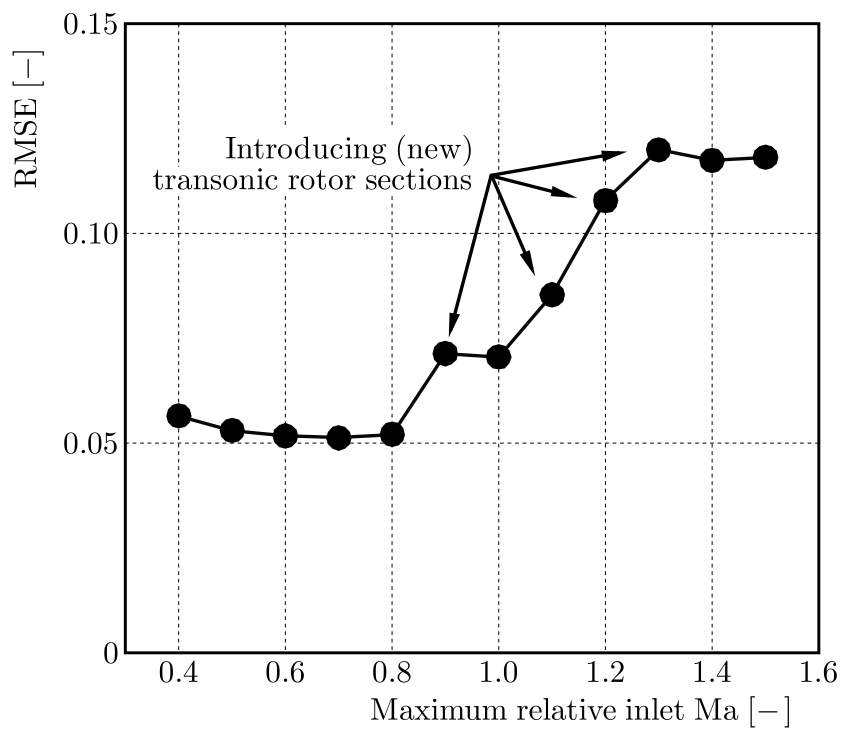

Fig. 6. RSME of the linear regression between the normalized $f(\delta)$ and incidence with restrictions on the maximum relative inlet $\mathrm{Ma}$ of the test conditions

gives the RMSE of the linear regression between the normalized $f(\delta)$ and incidence with the restriction on the maximum relative inlet Ma of the test conditions. The data points are collected from compressor test recordings including the transonic rotor sections of cases 4 to 7 in Table 1 . When the relative inlet $\mathrm{Ma}$ is smaller than 0.8, the RMSE keeps at around 0.05. The RMSE increases drastically when transonic rotor sections are introduced. The RMSE continues to grow with more transonic rotor sections added to the regression. The scope of this study is to identify the critical Ma of the linear correlation. The linear correlation between the incidence and $f(\delta)$ can be used without Ma modification when the relative inlet Ma is smaller than 0.8.

In this way, the incidence in Eq. (3.1) 2 can be replaced by $f(\delta)$. The most benefit is that both the loss, Eq. (3.5), and deviation angle, Eq. (3.6) $)_{1}$, can be predicted as long as $f(\delta)$, Eq. $(3.6)_{2}$, is calculated 


$$
\frac{\omega}{\omega_{\text {des }}}=\left|\sqrt[n]{\frac{c_{m}}{\omega_{\text {des }}}} \frac{f(\delta)-f(\delta)_{\text {des }}}{d f(\delta) / d i}\right|^{n}+1
$$

and

$$
\delta=\arctan \left[\tan \left(\kappa_{1}+i\right)-\frac{\sigma}{2 \cos \beta_{m}} f(\delta)\right]-\kappa_{2} \quad f(\delta)=\frac{d f(\delta)}{d i}\left(i-i_{\text {des }}\right)+f(\delta)_{\text {des }}
$$

The loss and deviation are the most important empirical parameters in the compressor performance predictions (Denton, 2017). When the blade design performance is known, the unknown values in Eqs. (3.5) and (3.6) are $c_{m}$ and $d f(\delta) / d i$. An empirical method is given to calculate $c_{m}$, and an analytical method is given to calculate $d f(\delta) / d i$. Both methods are derived from the blade design performance. The influence of the design performance on the off-design loss, and the correlation between loss and deviation are, therefore, given through the introduction of $f(\delta)$.

An empirical correlation, Eq. (3.7) 1 , is used to calculate the normalized $c_{m}$. The correlation is built through principal component analysis (PCA) based regressions. The design blade loading factor, design loss, solidity and the blade maximum thickness to chord ratio are the dominant factors that decide on the off-design loss changing rate empirically. Figure 7 shows the variation of $F\left(c_{m}\right)$ against $f(\delta)_{\text {des }}$ using Eq. (3.7) $)_{2}$ through formula transformation of Eq. $(3.7)_{1}$ when $n$ equals to 3

$$
\begin{aligned}
& \sqrt[n]{\frac{c_{m}}{\omega_{\text {des }}}}=\frac{\omega_{\text {des }}}{f(\delta)_{\text {des }} \sigma\left(10 t_{\text {max }} / c\right)}\left[c^{(1)} f(\delta)_{d e s}+c^{(0)}\right]^{2} \\
& F\left(c_{m}\right)=\sqrt[n]{\sqrt[n]{\frac{c_{m}}{\omega_{\text {des }}}} \frac{\left.f(\delta)_{\text {des }} \sigma\right)}{\omega_{\text {des }}} \frac{10 t_{\text {max }}}{c}}=c^{(1)} f(\delta)_{\text {des }}+c^{(0)}
\end{aligned}
$$

The empirical parameters $c^{(0)}$ and $c^{(1)}$ in Eq. $(3.7)_{1}$ are given for a positive real number index $n$

$$
\begin{aligned}
& c(1)= \begin{cases}\frac{1}{n}\left(7.871 \cdot 10^{-1}\right)+1.312 & \text { compressors } \\
\frac{1.342}{n}+1.326 & \text { cascades }\end{cases} \\
& \left(\frac{c^{(0)}}{c^{(1)}}\right)^{2}= \begin{cases}\frac{1}{n}\left(-1.013 \cdot 10^{-1}\right)+1.046 \cdot 10^{-1} & \text { compressors } \\
\frac{1}{n}\left(-5.204 \cdot 10^{-1}\right)+3.466 \cdot 10^{-1} & \text { cascades }\end{cases}
\end{aligned}
$$

The unknown slope $d f(\delta) / d i$ is calculated through analytical derivation

$$
\frac{d f(\delta)}{d i}=\frac{\partial f(\delta)}{\partial i}+\frac{\partial f(\delta)}{\partial \delta} \frac{d \delta}{d i}
$$

Special treatment of the term $d \delta / d i$ is required because the function between the deviation and the incidence is not defined. The definition of $f(\delta)$ Eq. (1.1) $)_{1}$, and the empirical linear correlation, Eq. (3.6) 2 , are combined to construct an implicit function between the incidence and deviation $\delta(i)$

$$
\delta(i): \quad \frac{2}{\sigma}\left[\tan \left(\kappa_{1}+i\right)-\tan \left(\kappa_{2}+\delta\right)\right] \cos \beta_{m}=\frac{d f(\delta)}{d i}\left(i-i_{\text {des }}\right)+f\left(\delta_{\text {des }}\right)
$$




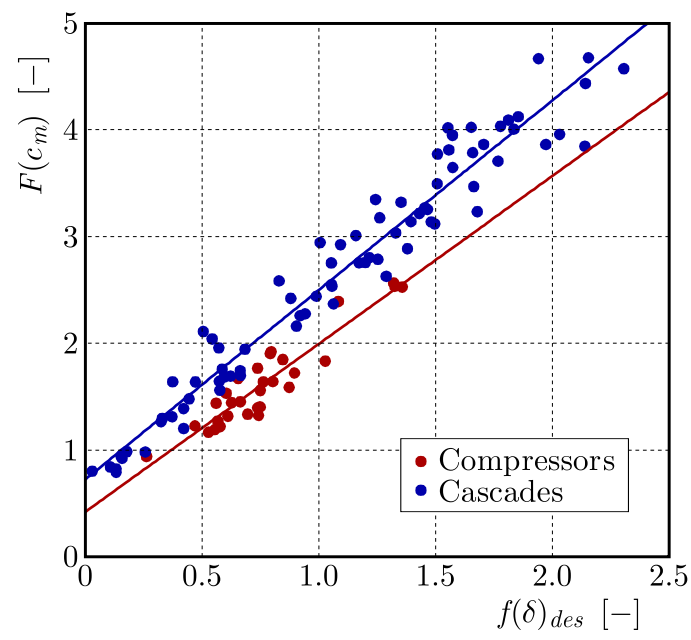

Fig. 7. Variation of the function of the off-design loss coefficient $F\left(c_{m}\right)$ with the blade design loading factor $f(\delta)_{\text {des }}$

Equations (3.11) are derived explicitly. Equation (3.12) is obtained by differentiation of the incidence $i$ terms on both sides of Eq. (3.10). The explicit form of the derivation terms are

$$
\begin{aligned}
& \frac{\partial f(\delta)}{\partial i}=\frac{2\left[\tan ^{2}\left(\kappa_{1}+i_{\text {des }}\right)+1\right]}{\sigma \sqrt{\tan ^{2} \beta_{m, d e s}+1}} \\
&- \frac{\left[\tan \left(\kappa_{1}+i_{\text {des }}\right)-\tan \left(\kappa_{2}+\delta_{\text {des }}\right)\right]\left[\tan ^{2}\left(\kappa_{1}+i_{\text {des }}\right)+1\right] \tan \beta_{m, d e s}}{\sigma \sqrt{\left(\tan ^{2} \beta_{m, d e s}+1\right)^{3}}} \\
& \frac{\partial f(\delta)}{\partial \delta}=-\frac{2\left[\tan ^{2}\left(\kappa_{2}+\delta_{\text {des }}\right)+1\right]}{\sigma \sqrt{\tan ^{2} \beta_{m, d e s}+1}} \\
&-\frac{\left[\tan \left(\kappa_{1}+i_{\text {des }}\right)-\tan \left(\kappa_{2}+\delta_{\text {des }}\right)\right]\left[\tan ^{2}\left(\kappa_{2}+\delta_{\text {des }}\right)+1\right] \tan \beta_{m, d e s}}{\sigma \sqrt{\left(\tan ^{2} \beta_{m, d e s}+1\right)^{3}}}
\end{aligned}
$$

and

$$
\begin{aligned}
\frac{d \delta}{d i} & =\frac{\left[\tan ^{2}\left(\kappa_{1}+i_{\text {des }}\right)+1\right]\left[\tan ^{2}\left(\kappa_{2}+\delta_{\text {des }}\right)+\tan \left(\kappa_{1}+i_{\text {des }}\right) \tan \left(\kappa_{2}+\delta_{\text {des }}\right)+2\right]}{\left[\tan ^{2}\left(\kappa_{2}+\delta_{\text {des }}\right)+1\right]\left[\tan ^{2}\left(\kappa_{1}+i_{\text {des }}\right)+\tan \left(\kappa_{1}+i_{\text {des }}\right) \tan \left(\kappa_{2}+\delta_{\text {des }}\right)+2\right]} \\
& -\frac{\frac{d f(\delta)}{d i} \sigma \sqrt{\left(\tan ^{2} \beta_{m, d e s}+1\right)^{3}}}{\left[\tan ^{2}\left(\kappa_{2}+\delta_{\text {des }}\right)+1\right]\left[\tan ^{2}\left(\kappa_{1}+i_{\text {des }}\right)+\tan \left(\kappa_{1}+i_{\text {des }}\right) \tan \left(\kappa_{2}+\delta_{\text {des }}\right)+2\right]}
\end{aligned}
$$

The blade design performance is used in the calculation because $d f(\delta) / d i$ keeps constant at all operating conditions of the blade profile. Iteration is required to solve Eq. (3.9) because $d f(\delta) / d i$ also presents in Eq. (3.12). To initialize the iteration, $d f(\delta) / d i$ can be replaced by the partial derivation term $\partial f(\delta) / \partial i$.

Figure 8 shows the comparison of the predicted $d f(\delta) / d i$ with the experimental values. The values of $d f(\delta) / d i$ are generally larger for cascade tests with high setting angles because the tangential terms in $f(\delta)$ vary more rapidly at higher setting angles. For rotors, the setting angles are smaller than $50^{\circ}$. For stators, the setting angles are smaller than $25^{\circ}$. The predicted values are in good agreement with the experimental values for compressors. For cascades, discrepancies occur at larger $d f(\delta) / d i$ conditions. There is also a trend that the slope is under-predicted when the setting angle is extremely high $\left(60^{\circ}\right.$ or $\left.70^{\circ}\right)$ in the cascade tests. Further investigation is needed to give a better solution for those extreme cases. Figure 9 gives the probability plot of 
the off-design loss prediction error using the $f(\delta)$ based prediction method. The data points are collected from Tables 1 and 2. The maximum error is smaller than 0.08 . For $90 \%(0.05,0.95)$ of the data points, the prediction error is in the margin of \pm 0.02 . Considering the uncertainty of the pressure measurement, the margin of the prediction error is relatively small.

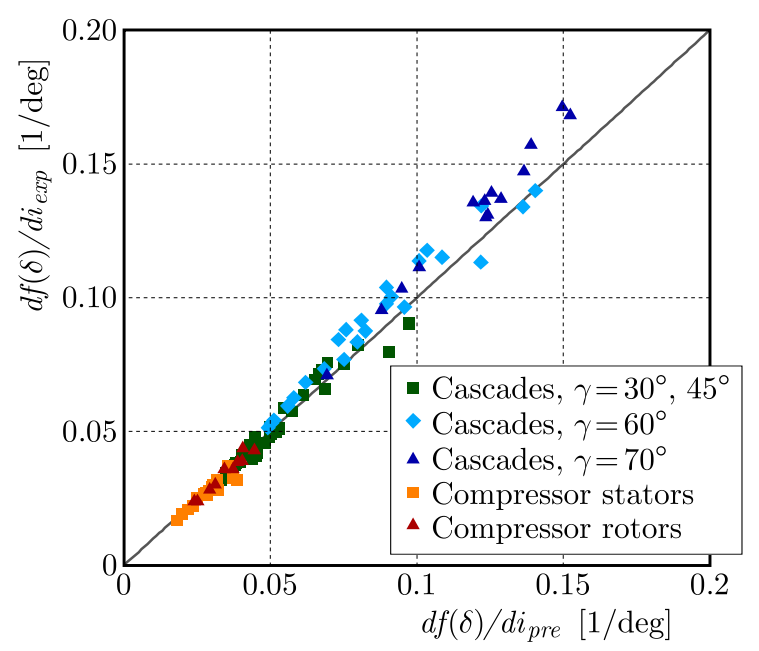

Fig. 8. Comparison of the predicted $d f(\delta) / d i$ with the experimental values

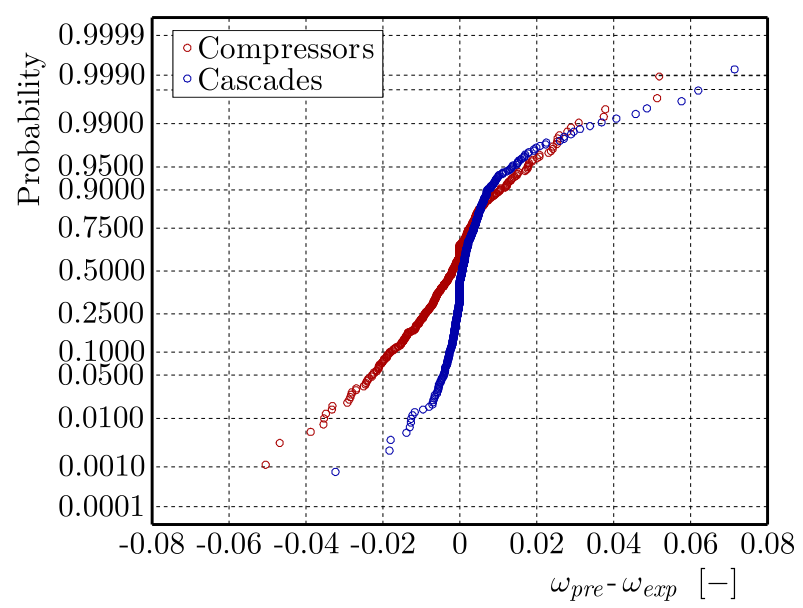

Fig. 9. Probability plot of the off-design loss prediction error using the $f(\delta)$ based prediction method

\section{Validation of the prediction method}

The off-design loss prediction method is firstly validated for cascades. Figure 10 shows the normalized off-design loss cloud map of NACA65 series cascade tests with 6 percent maximum thickness to chord. With the cloud map, the design space of the off-design incidence is shown clearly for the same series of blade design. The colour contour shows the experimental results. The solid isolines are the predicted normalized losses. The loss is usually slightly under-predicted when the normalized loss is greater than 2 because linear extrapolation is used outside of its designated range of application (where $\omega / \omega_{\text {des }}<2$ ) in practice. The purpose is to reduce the numerical oscillation during the design iterations.

For compressors, the off-design loss prediction is incorporated into a streamline curvature method solver based primarily on Denton's (1978) method. The loss is predicted at different 


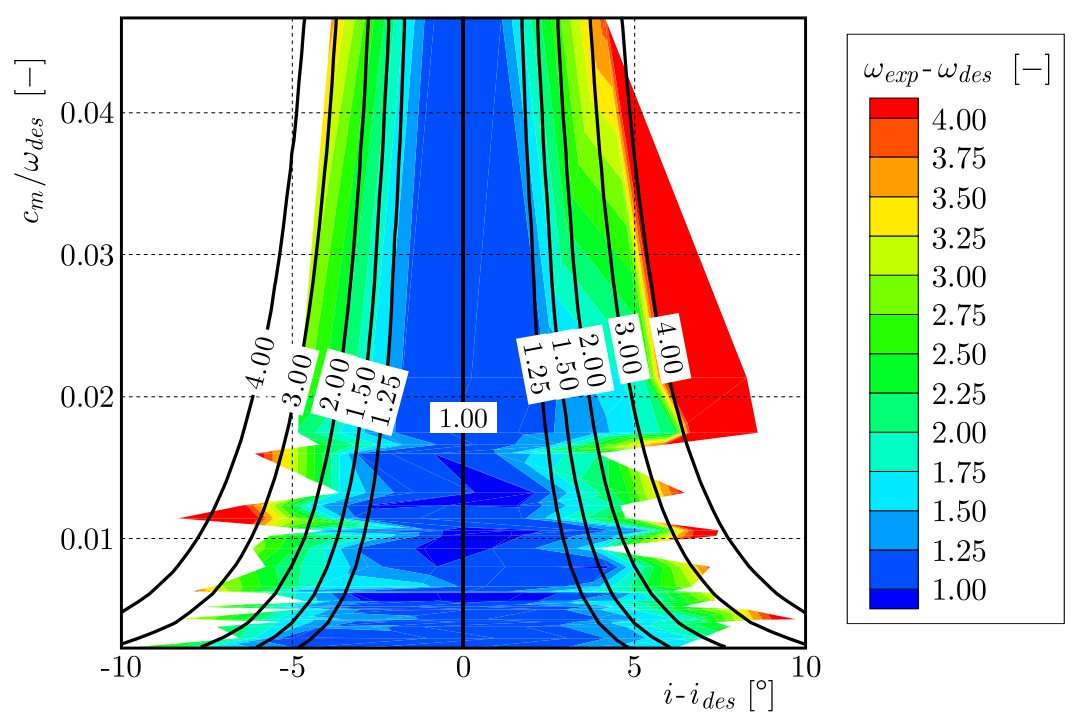

Fig. 10. Normalized off-design loss cloud map of NACA65 cascade tests with 6 percent maximum thickness to chord
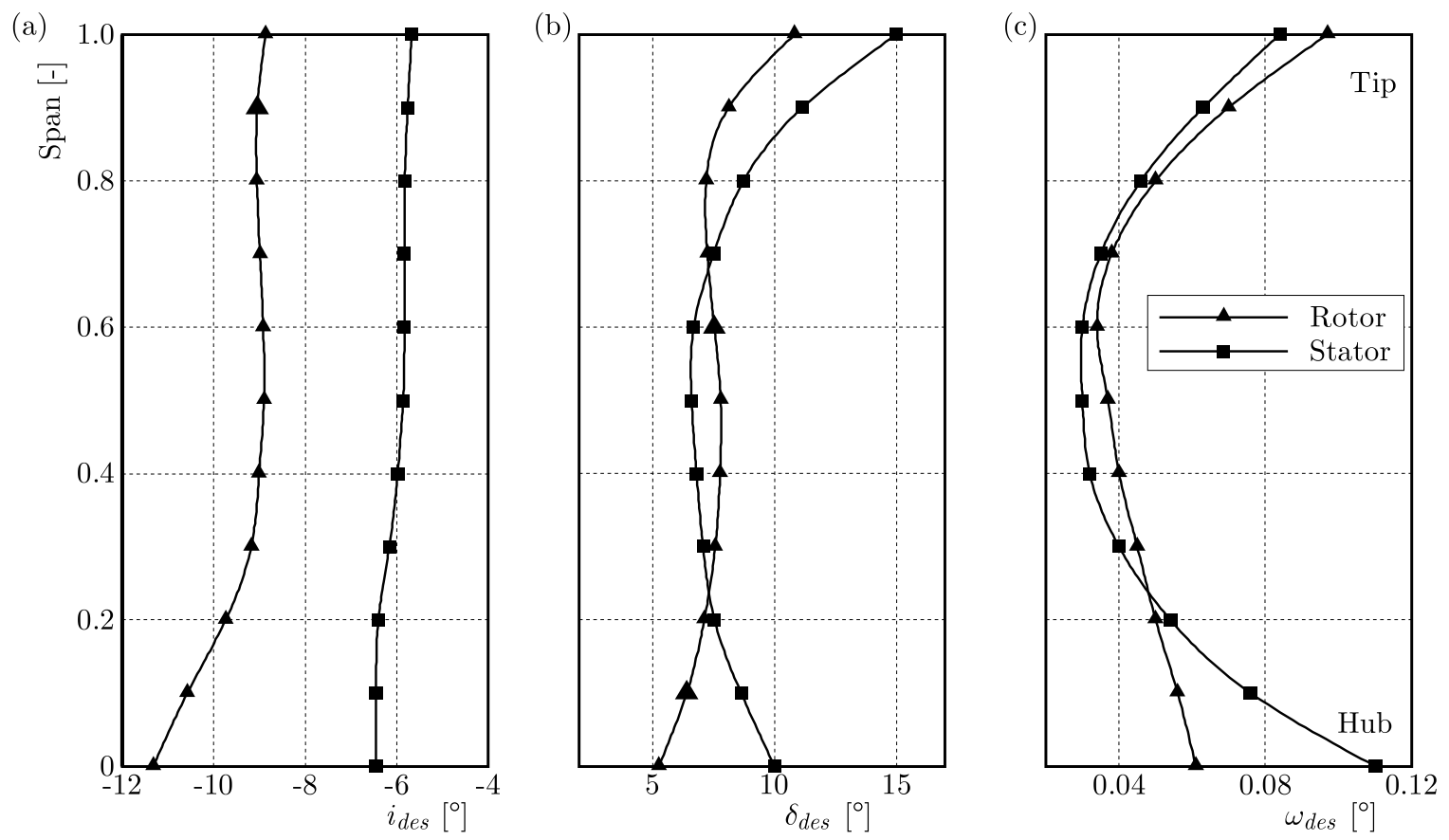

Fig. 11. Design incidence (a), deviation (b), and loss (c) of rotors and stators in the LSRC

span sections individually and smoothed along the spanwise direction using the adjacent points. For brevity, no other spanwise distribution model is introduced in the performance prediction. The test recordings of the four stage GE E3 LSRC are studied. The input of the off-design loss prediction is the blade design incidence, deviation, and loss. Figure 11 gives the blade design performance of the rotors and stators in the LSRC collected from the blade design report (Wisler, 1977). The four stages have identical geometries and the same inputs. Figure 12 gives the isentropic efficiency (a) and static pressure rise (b) character lines of the LSRC. The predicted character lines are generally in good agreement with the experimental values. In Figure 13, the spanwise distribution of losses is investigated at design point (DP) and near stall (NS) conditions in detail. The total pressure loss coefficients determined from the relative total pressure measurements and the absolute total pressure measurements in the third stage are used to compare 


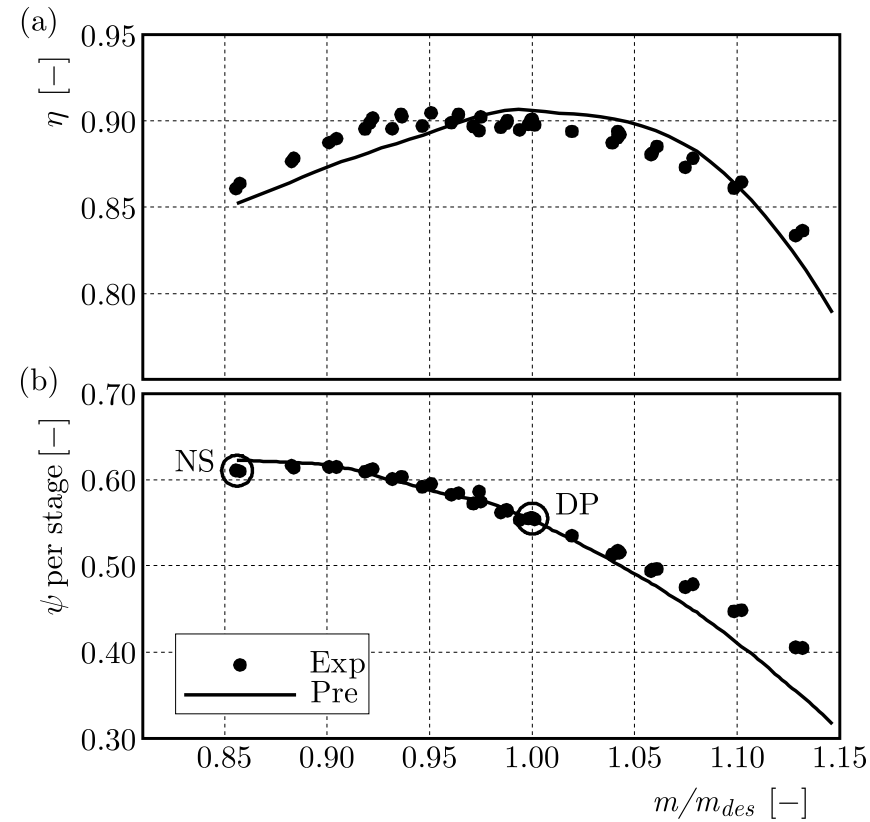

Fig. 12. Isentropic efficiency (a) and static pressure rise (b) character lines of the LSRC

(a)

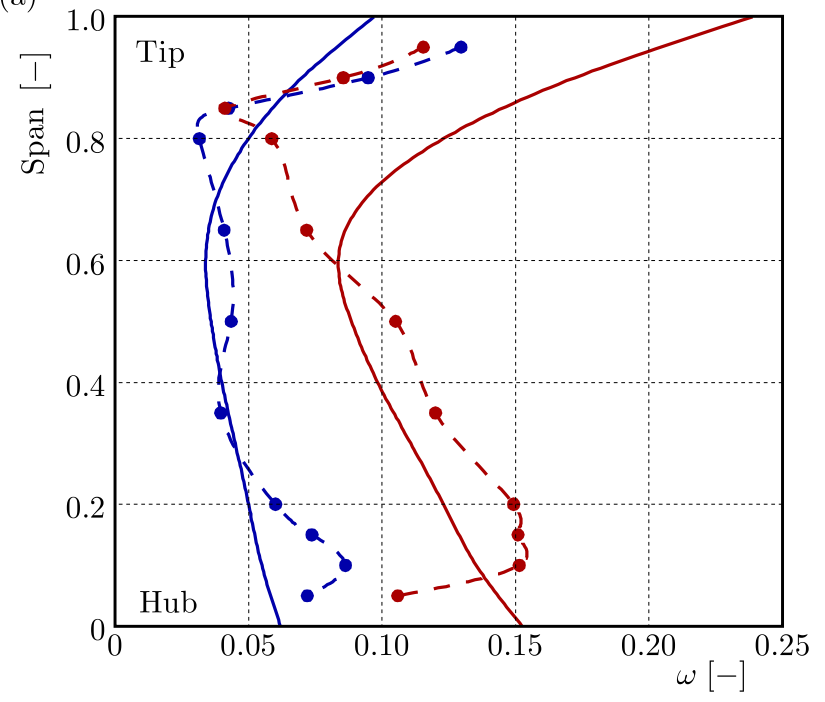

(b)

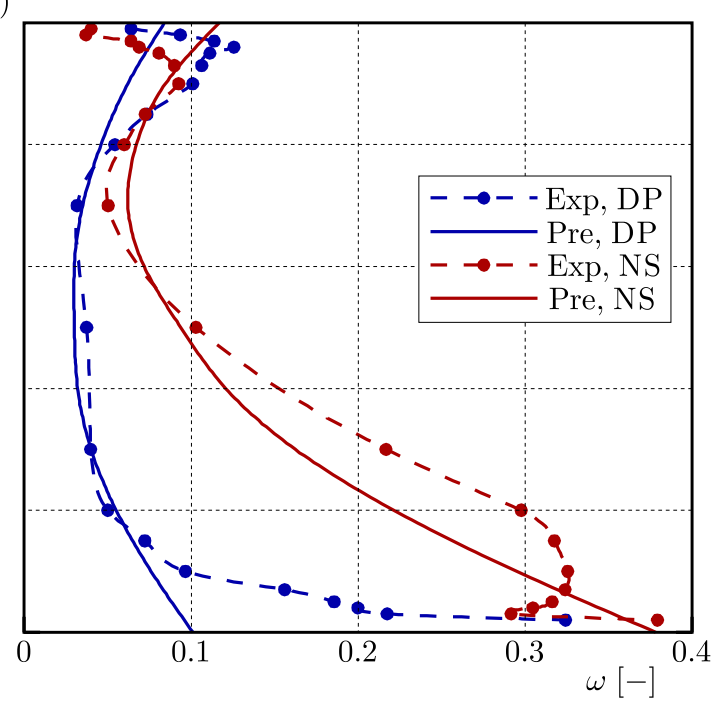

Fig. 13. Comparison of the spanwise distributions of the predicted and experimental losses at DP and NS conditions for the third stage rotor (a) and stator (b) of the LSRC

with the predicted values. At DP condition, the loss outside the endwall region (30\% to $70 \%$ span) is well predicted, while the loss of the endwall flow is under-estimated. The loss prediction is not modified for the endwall flow. The loss of the endwall flow is usually larger at DP condition. At NS condition, the loss prediction is challenging. The performance deterioration is mostly captured by the prediction model, but the rotor tip loss is exaggerated in the prediction. In the experiment, the difference of the rotor loss is little above the $85 \%$ span. Also, the increase of the loss near the endwall is over-predicted. The discrepancy indicates two major issues to be noted in the further modification. Firstly, the endwall and the rotor tip losses are generally larger than their freestream span design values at their designed operating condition. Secondly, the variation of the endwall and the rotor tip losses can be small when compared to the variation of the freestream span losses. The modification of the endwall flows will be addressed with more systematic experimental cases in the further study. 


\section{Conclusions}

A blade performance based off-design loss prediction method is given through the introduction of the incidence based polynomial prediction function and the linear correlation between incidence and $f(\delta)$ with AVDR modification. The method is applicable when the relative inlet Ma is smaller than 0.8. The method shows the influence of blade design performance on the off-design loss variation empirically. The method also gives an empirical correlation between the deviation angle and loss at off-design conditions.

Multiple series of compressors and cascades are used to demonstrate the correlation in the prediction method. It has been shown that the design blade loading factor, design loss, solidity, and blade maximum thickness to chord ratio are the dominant factors that decide on the off-design loss changing rate empirically. It has been also shown that the analytical derivation of the changing rate of $f(\delta)$ against the incidence can be calculated by incorporating the statistical linear correlation in the derivation.

In application, it has been shown that the prediction method can be used to demonstrate the design space of the off-design incidence for a blade series. The prediction method is reliable in the compressor character line and performance prediction. For further improvement, the modification of the endwall and the rotor tip flows is a feasible way to increase the accuracy of the current prediction method.

\section{Acknowledgements}

This work was supported by the Natural Science Foundation of China (No. 51576124), the Research Program of Shanghai Municipal Commission of Economy and Informatization (No. CXY-2016-005), and the United Innovation Center (UIC) of Aerothermal Technologies for Turbomachinery.

\section{References}

1. Aungier R.H., 2003, Axial Flow Compressors: A Strategy for Aerodynamic Design and Analysis, ASME Press, New York, p. 131

2. Banjac M., Petrovic M.V., 2018, Development of method and computer program for multi-stage axial compressor design: Part II - Two-dimensional design and validation using CFD, ASME Proceedings, GT2018-75412

3. Boyer K.M., O'Brien W.F., 2002, An improved streamline curvature approach for off-design analysis of transonic axial compression systems, ASME Proceedings, GT2002-30444

4. Cetin M., Ucer A.S., Hirsch C., Serovy G.K., 1987, Application of modified loss and deviation correlations to transonic axial compressors, AGARD Report, $\mathbf{7 4 5}$

5. Cumpsty N.A., 2004, Compressor Aerodynamics, 2nd ed. Krieger Publishing Company, p. 137-140

6. Denton J.D., 1978, Throughflow calculations for transonic axial-flow turbines, Journal of Engineering for Power, 100, 212-218

7. Denton J.D., 2017, MULTALL - an open source, CFD based, turbomachinery design system, ASME Proceedings, GT2017-63993

8. EMERY J.C., 1957, Low-speed cascade investigation of thin low-camber NACA 65-series blade sections at high inlet angles, NACA Report, RML57E03

9. Herrig L.J., Emery J.C., Erwin J.R., 1951, Systematic two-dimensional cascade tests of NACA 65-series compressor, NACA Report, TR1368

10. Jasen W., Moffatt W.C., 1967, The off-design analysis of axial-flow compressors, Journal of Engineering for Power, 89, 453-462 
11. König W.M., Hennecke D.K., Fottner L., 1996, Improved blade profile loss and deviation angle models for advanced transonic compressor bladings: Part I - A model for subsonic flow, Journal of Turbomachinery, 118, 73-80

12. Leggett J., Priebe S., Shabbir A., Michelassi V., Sandberg R., Richardson E., 2018, Loss prediction in an axial compressor cascade at off-design incidences with free stream disturbances using large eddy simulation, Journal of Turbomachinery, 140, 071005

13. Lewis G.W. JR., TysL E.R., 1974, Overall and blade-element performance of a 1.20-pressure-ratio fan stage at design blade setting angle, NASA Report, TMX3101

14. Lewis G.W. JR., Reid L., TysL E.R., 1974, Design and performance of a high-pressure-ratio, highly loaded axial-flow transonic compressor stage, NASA Report, TMX3100

15. Moore R.D., REID L., 1980, Performance of single-stage axial-flow transonic compressor with rotor and stator aspect ratios of 1.19 and 1.26, respectively, and with design pressure ratio of 2.05, NASA Report, TP1659

16. Moore R.D., Reid L., 1982a, Performance of single-stage axial-flow transonic compressor with rotor and stator aspect ratios of 1.63 and 1.78, respectively, and with design pressure ratio of 1.82 , NASA Report, TP1974

17. Moore R.D., Reid L., 1982b, Performance of single-stage axial-flow transonic compressor with rotor and stator aspect ratios of 1.63 and 1.77, respectively, and with design pressure ratio of 2.05, NASA Report, TP2001

18. Osborn W.M., Moore R.D., Steinke R.J., 1978, Aerodynamic performance of a 1.35-pressure-ratio axial-flow fan stage, NASA Report, TP1299

19. Pollard D., Gostelow J.P., 1967, Some experiments at low speed on compressor cascades, Journal of Engineering for Gas Turbines and Power, 89, 427-436

20. Reid L., Moore R.D., 1978, Performance of single-stage axial-flow transonic compressor with rotor and stator aspect ratios of 1.19 and 1.26, respectively, and with design pressure ratio of 1.82 , NASA Report, TP1338

21. Schnoes M., Nicke E., 2015, Automated calibration of compressor loss and deviation correlations, ASME Proceedings, GT2015-42644

22. Schnoes M., Nicke E., 2017, A database of optimal airfoils for axial compressor throughflow design, Journal of Turbomachinery, 139, 051008

23. SWAN W.C., 1961, A practical method of predicting transonic-compressor performance, Journal of Engineering for Gas Turbines and Power Power, 83, 322-330

24. Urasek D.C., Gorrell W.T., Cunnan W.S., 1979, Performance of two-stage fan having low-aspect-ratio, first-stage rotor blading, NASA Report, TP1493

25. Wisler D.C., 1977, Core compressor exit stage study. Vol. I - Blading design, NASA Report, CR135391

26. Wisler D.C., 1980, Core compressor exit stage study. Vol. II - Data and performance report for the baseline configurations, NASA Report, CR159498

27. Wu D., Teng J., Qiang X., Feng J., 2017, An implicit off-design deviation angle correlation of axial flow compressor blade elements, ASME Proceedings, GT2017-63189

28. Zhang L., Congiu F., Gan X., Karunakara D., 2017, Performance prediction and optimization of low pressure steam turbine radial diffuser at design and off-design conditions using streamline curvature method, Journal of Engineering for Gas Turbines and Power, 139, 072601-1 